\title{
Seismicity Alert Probabilities at Parkfield, California, Revisited
}

\author{
by Andrew J. Michael and Lucile M. Jones
}

\begin{abstract}
For a decade, the U.S. Geological Survey has used the Parkfield Earthquake Prediction Experiment scenario document to estimate the probability that earthquakes observed on the San Andreas fault near Parkfield will turn out to be foreshocks followed by the expected magnitude 6 mainshock. During this time, we have learned much about the seismogenic process at Parkfield, about the long-term probability of the Parkfield mainshock, and about the estimation of these types of probabilities. The probabilities for potential foreshocks at Parkfield are reexamined and revised in light of these advances. As part of this process, we have confirmed both the rate of foreshocks before strike-slip earthquakes in the San Andreas physiographic province and the uniform distribution of foreshocks with magnitude proposed by earlier studies. Compared to the earlier assessment, these new estimates of the long-term probability of the Parkfield mainshock are lower, our estimate of the rate of background seismicity is higher, and we find that the assumption that foreshocks at Parkfield occur in a unique way is not statistically significant at the $95 \%$ confidence level. While the exact numbers vary depending on the assumptions that are made, the new alert probabilities are lower than previously estimated. Considering the various assumptions and the statistical uncertainties in the input parameters, we also compute a plausible range for the probabilities. The range is large, partly due to the extra knowledge that exists for the Parkfield segment, making us question the usefulness of these numbers.
\end{abstract}

\section{Introduction}

The quest to turn seismological research results into socially important products can be a difficult process when scientific uncertainty must be translated into public action. This article illustrates this process by detailed examination of one example: the calculation of the probability that an earthquake near Parkfield, California (Fig. 1), is a foreshock to the expected magnitude 6 mainshock on the San Andreas fault. These probabilities for potential foreshocks are by far the best constrained of all the Parkfield alert probabilities. They are, in fact, the ad hoc basis for the rest of the possible alerts such as those based on surficial fault creep, strainmeter, or water-well data (Bakun et al., 1987, hereafter referred to as OFR 87-192). Moreover, we know more about previous foreshocks and mainshocks at Parkfield than along any other fault segment. Despite, or perhaps because of, this extra knowledge, we will demonstrate that the foreshock alert probabilities have considerable uncertainty.

The results in this article do not represent testable science, at least not over any practical timescale. We demonstrate in this article what is needed to make meaningful statements of earthquake probabilities. Because that information is not yet available, we have not, and indeed cannot, make verifiable statements of the actual probabilities. We rather demonstrate the difficulties and pitfalls involved with turning scientific results into a socially useful product.
The Parkfield Earthquake Prediction Experiment scenario document (OFR 87-192) includes estimates of the probability that earthquakes observed in the Parkfield area, and therefore possible foreshocks, will be followed by the magnitude 6 Parkfield mainshock. It also takes these probabilities and relates them to alert levels A through $\mathrm{D}$ that can be used to summarize the experiment's status. This system has been used since before the publication of OFR 87-192, including A-level alerts in October 1992 and November 1993.

The probability estimates in OFR $87-192$ should be reevaluated for three reasons. First, these probabilities depend on the long-term probability of the mainshock occurring. In OFR 87-192, this was based on the model of Bakun and Lindh (1985) and included the prediction that the mainshock would occur before 1993 with $95 \%$ confidence. This prediction was not fulfilled, and therefore, the probabilities should be reevaluated.

Second, simply because time has passed and the seismographic network has improved, we can use better data to determine the rate of background seismicity. Moreover, OFR 87-192 limits the area in which the higher-level alerts can occur based on the observations of foreshocks in 1934 and 1966. Based on the experience of the past few years, there 


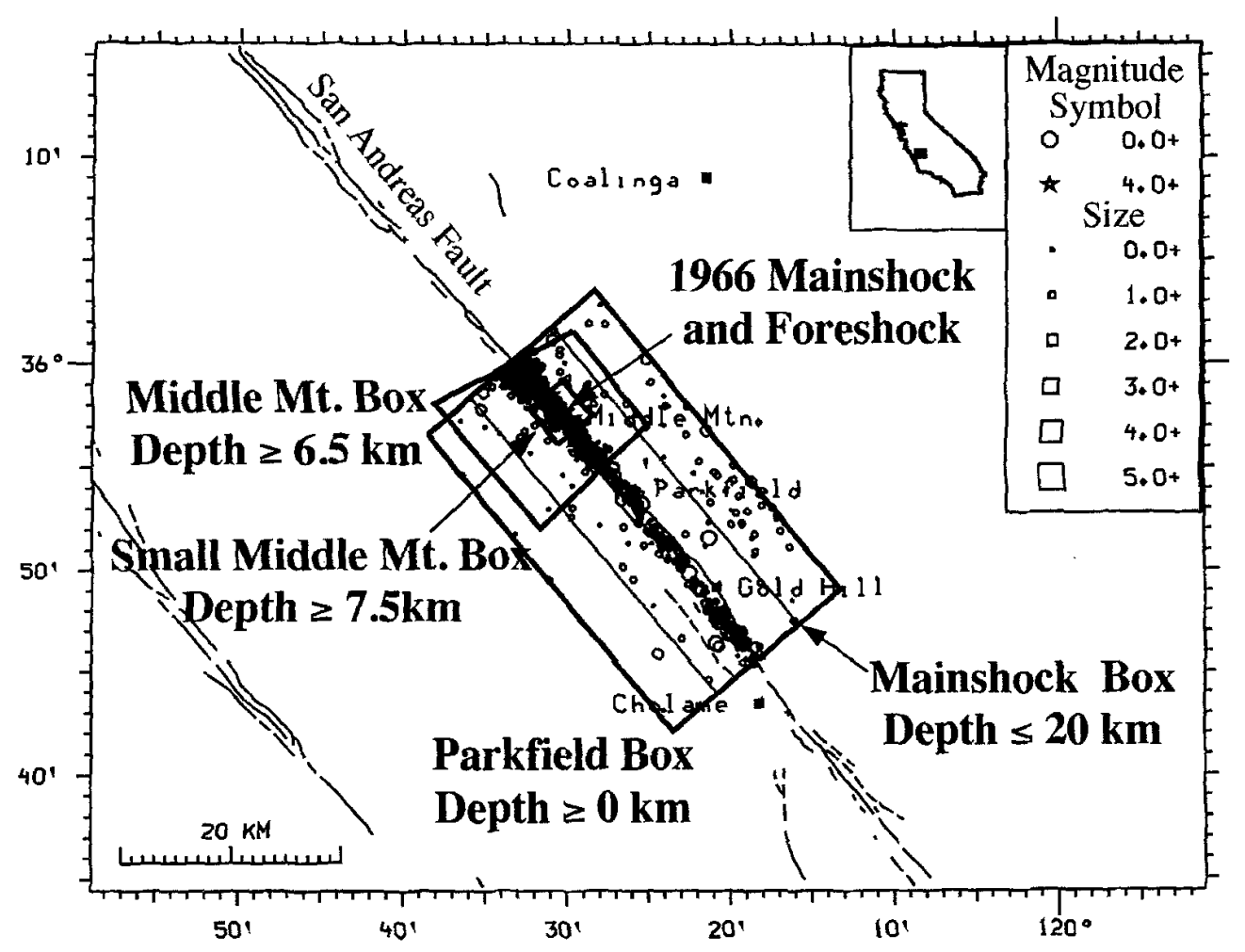

Figure 1. Map showing the background seismicity at Parkfield from 1982 through 1994, three possible boxes on which to base the alert structure, and a box defined to contain the predicted mainshock hypocenter.

are arguments both to restrict further this area and to relax greatly these restrictions that should be considered.

Third, since the time of writing of OFR 87-192, the methods for computing these types of probabilities have improved (Agnew and Jones, 1991). Some subtle inconsistencies in the mathematical approach used in OFR 87-192 to compute these probabilities have been revealed ( $\mathrm{Lindh}$ and Lim, 1995; Michael and Jones, 1995). For instance, although an attempt was made to correlate the Parkfield foreshocks with the average rate in California, the rate of large foreshocks assumed in OFR 87-192, based on the events in 1901, 1922, 1934, and 1966, is three times as high at large magnitudes as that observed on strike-slip faults throughout the San Andreas physiographic province (as defined by Zoback and Zoback, 1980).

To complete our reevaluation of the Parkfield alert probabilities, we must reevaluate (1) the rate of foreshocks before mainshocks as a function of magnitude and the appropriate mathematical form to express that, (2) the appropriate alert area (possible location for the foreshock), and (3) the longterm probability of the mainshock. Evaluating each of these factors requires making assumptions, and each assumption has some effect on the results. We will examine the basis for each assumption and their effect on the results. Our final result will be a preferred set of assumptions, the foreshock probabilities that follow from them, and a range of plausible probabilities based on the full range of assumptions.
Method

Agnew and Jones (1991) considered the general problem of deriving the probability that the characteristic earthquake on a fault will occur after a smaller earthquake near that fault, either a background earthquake or a foreshock, has occurred. Their method is an improvement over OFR $87-$ 192, which contained some subtle inconsistencies (Lindh and Lim, 1995; Michael and Jones, 1995).

Agnew and Jones (1991) assumed that the earthquakes of interest could be divided into three classes: background events, foreshocks, and mainshocks. By this classification, foreshocks are always followed by mainshocks, and background events are never followed by mainshocks. In their system, mainshocks are identified by reaching a magnitude threshold for a given fault segment. But there is no way to determine whether other events are background events or a foreshocks, except to wait to see if the mainshock occurs. Then the answer is of little practical use. We can, however, determine the probability that a candidate event is a foreshock and that therefore the mainshock will follow. We call this the alert probability.

Agnew and Jones (1991) showed that if

$P(C)=$ probability of the characteristic mainshock at a given time, $P(B)=$ probability of a background earthquake, 
$P(F)=$ probability of a foreshock,

$P(C \mid F \cup B)=$ probability of a characteristic mainshock given an event that may be either a foreshock or a background event, and

$P(F \mid C)=$ probability of a foreshock given a mainshock,

then

$P(C \mid F \cup B)=P(F) /[P(F)+P(B)]$.

Thus the probability that an event is a foreshock is the ratio of foreshocks to total events: foreshocks and background events. We can measure the rate of background earthquakes and estimate $P(B)$, but $P(F)$ cannot be measured because there is too little data. Instead, Agnew and Jones used

$$
P(F)=P(F \mid C) P(C)
$$

or that the probability of a foreshock is the probability of the mainshock times the probability that a mainshock is preceded by a foreshock. This yields

$$
P(C \mid F \cup B)=P(F \mid C) P(C) /[P(F \mid C) P(C)+P(B)]
$$

It satisfies common sense because if either the probability of the mainshock or the percentage of mainshocks preceded by foreshocks $[P(C)$ or $P(F \mid C)]$ is zero, so is the alert probability. Also, if all nonmainshocks are foreshocks $[P(B)=$ $0]$, the probability is 1 . Thus, the probability that an earthquake will be a foreshock can be calculated when we know the background probability for that earthquake $P(B)$, the probability of having the Parkfield mainshock independent of any potential foreshocks, $P(C)$, and the probability that a foreshock precedes the mainshock $P(F \mid C)$.

\section{Input Parameters}

This section examines the three input parameters for the alert probability calculation. Determining each requires making a number of assumptions. These assumptions, and statistical errors in determining various quantities, will have an effect on the results. Thus, each requires close examination.

\section{Mainshock Probability}

The OFR 87-192 alert probabilities used a long-term probability for the mainshock of $15 \%$ per year. This was based on the Bakun and Lindh (1985) model at the beginning of 1986. Applying their model to the current time would result in an even larger annual probability (OFR 87-192). The failure of the Parkfield mainshock to occur during the $95 \%$ confidence window predicted by Bakun and Lindh (1985) has invalidated that model and requires selecting a different model. A review of the Parkfield Earthquake Prediction Experiment (NEPEC Working Group, 1993) under the auspices of the National Earthquake Prediction Evaluation Council concluded that a variety of models suggest that the annual probability that the Parkfield mainshock will occur is around $10 \%$. The models discussed in the NEPEC report are purely statistical analyses of the sequence of six events that occurred in 1857, 1881, 1901, 1922, 1934, and 1966. These models use a variety of distributions that all assume some sort of semi-periodic behavior. Another alternative is the Poisson model that would lower the annual probability of the mainshock occurring to $4 \%$. The semi-periodic nature of the Parkfield mainshocks cannot yet be rejected (Savage, 1993), and we will follow the NEPEC Working Group in choosing it as the preferred model. However, we will consider the Poisson model when computing the range of possible alert probabilities.

Choosing higher or lower values for the long-term probability would increase or decrease the output alert probabilities, respectively. Doubling or halving the long-term probability would approximately double or halve the alert probabilities, respectively. This approximation holds as long as it is much more likely that a candidate event is a background earthquake and not a foreshock.

\section{Foreshock Rate}

For strike-slip earthquakes in California, Agnew and Jones (1991) assumed a distribution in which half of all $M \geqq 5$ mainshocks are preceded by one or more $M \geqq 2$ foreshocks within $10 \mathrm{~km}$ and 3 days, based on the results of Jones (1984). Including the 1966 Parkfield mainshock and foreshock sequence, Jones (1984) studied 16 strike-slip mainshocks with $M \geqq 5$, from 1966 to 1980 , within the San Andreas physiographic province as defined by Zoback and Zoback (1980). Seven of the sixteen sequences had $M \geqq 2$ foreshocks within the three days preceding the mainshock and within $10 \mathrm{~km}$ of the mainshock.

To update this, we identified 17 additional strike-slip sequences with $M \geqq 5$ mainshocks through the end of 1994 . Of these, 10 have $M \geqq 2$ foreshocks within three days of origin time (Table 1, Fig. 2a). This gives a total of 17 of 33 sequences that have foreshocks, which is extremely close to the original result of Jones (1984), and therefore, there is no reason to update the overall foreshock rate used in Agnew and Jones (1991).

For the magnitude distribution of foreshocks with respect to the mainshock, Agnew and Jones (1991) used a uniform distribution; so that any equal-sized interval in magnitude, less than the mainshock magnitude, has the same probability of containing a foreshock. This was based on an analysis of $669 M \geqq 3$ mainshocks with $M \geqq 2$ foreshocks recorded in southern California. Matching the data from Jones (1984), they show that the probability that any unit of magnitude will have the largest foreshock is $15 \%$. They also show that within 6.5 units of magnitude, all events should have a foreshock. Obviously, this uniform distribution has problems when using large magnitude differences, but this was judged not to be a practical problem. Applying this distribution to larger events like Parkfield requires a belief in 
Table 1

M $\geqq 5$ Strike-Slip Mainshocks, 1981-1994, in the San Andreas Physiographic Province and Their Foreshock Behavior

\begin{tabular}{cccccc}
\hline $\begin{array}{c}\text { Date } \\
\text { (yymmdd) }\end{array}$ & $\begin{array}{c}\text { Origin } \\
(\mathrm{h} \text { min sec) }\end{array}$ & $\begin{array}{c}\text { Lat } \\
\left({ }^{\circ} \mathrm{N}\right)\end{array}$ & $\begin{array}{c}\text { Long } \\
\left({ }^{\circ} \mathrm{W}\right)\end{array}$ & Mag* & Fore* \\
\hline 810426 & 120947.02 & 3305.91 & 11537.90 & 5.7 & 4.1 \\
810904 & 155050.13 & 3339.09 & 11905.58 & 5.5 & \\
840123 & 054000.00 & 3621.19 & 12154.51 & 5.2 & \\
840424 & 211500.00 & 3718.81 & 12139.39 & 6.2 & 2.3 \\
860126 & 192000.00 & 3648.56 & 12117.29 & 5.7 & \\
860331 & 115500.00 & 3728.02 & 12141.52 & 5.5 & 2.6 \\
861121 & 233300.00 & 4021.30 & 12425.63 & 5.1 & 2.3 \\
870207 & 034514.85 & 3223.28 & 11518.27 & 5.4 & 3.0 \\
870731 & 235600.00 & 4024.52 & 12424.43 & 5.5 & 3.2 \\
871124 & 131556.71 & 3300.87 & 11551.10 & 6.6 & 6.2 \\
880220 & 083900.00 & 3647.68 & 12118.65 & 5.1 & \\
881203 & 113826.44 & 3409.06 & 11807.81 & 5.0 & \\
900116 & 200800.00 & 4014.63 & 12423.04 & 5.4 & \\
900228 & 234336.75 & 3408.62 & 11741.84 & 5.4 & 3.7 \\
920423 & 045023.22 & 3357.67 & 11619.05 & 6.1 & 4.6 \\
920628 & 115734.13 & 3412.01 & 11626.20 & 7.3 & 3.6 \\
920711 & 181416.15 & 3512.60 & 11803.94 & 5.7 & \\
\hline
\end{tabular}

* Mag is the mainshock magnitude. Fore is the magnitude of a foreshock if there was an $M \geqq 2$ foreshock in the 3 days before the listed mainshock and within $10 \mathrm{~km}$ of the mainshock hypocenter.

the self-similarity of the foreshock process. They also noted that the form of the magnitude distribution of foreshocks was very uncertain.

Lindh and Lim (1995) suggested that the magnitude distribution of foreshocks with respect to the mainshock should be log-normal with a mean of 0.52 and a standard deviation of 0.62 . Their data set included $30 M \geqq 5$ mainshocks in a region similar to the San Andreas physiographic province. Slight differences between their data set and the one presented earlier are due to the different starting time, the magnitude chosen for specific events, and the exact region used.

Determining the difference between the uniform and log-normal distributions requires a data set large enough for reliable statistical analysis. The data set of 33 events presented above (Fig. 2a) and the 30 events presented by Lindh and Lim (1995, Fig. 2b) are both too small for this purpose. For instance, the data set from Lindh and Lim (1995) has three earthquakes with foreshocks within 1 magnitude unit of the mainshock and seven from one to two units from the mainshock. The uniform distribution would suggest that each of these bins have five events. Thus the difference between the uniform distribution and these data is only two events per bin. A Kolmogorov-Smirnoff one-sample test can be used to test the data versus each of the two proposed distributions. For the Lindh and $\operatorname{Lim}$ (1995) data, we divided the data into $2,3,4,5$, and 6 equal-sized bins. Regardless of the bin size, neither distribution could be rejected even at the $80 \%$ confidence level. Which distribution fits the data better changes frequently with the bin size, perhaps a symptom that with such a small data set the tests have little statistical power. We conclude that this data set is simply too small. The data set of 33 events presented earlier leads to virtually identical results.

The data set can be enlarged by including smaller-magnitude events, a longer-time period, and/or a larger area. Using smaller events risks problems with catalog completeness. Using a longer time period requires using mainshocks with unknown focal mechanisms in addition to increasing problems with catalog completeness. Using a larger area requires including different physiographic provinces that may have different seismogenic behaviors. Below we investigate the effects of using both longer time periods and smaller events but maintain the spatial restriction.

We searched the Caltech Southern California catalog back to 1933 and the U.C. Berkeley Northern California catalog back to 1950 for $M \geqq 5$ mainshocks in the San Andreas physiographic province. We then removed known dipslip events and determined the magnitude of the largest foreshock, if any existed. The resulting catalog of 97 events, 40 of which have foreshocks, is fraught with problems. First, it is likely to contain an unknown number of thrust events that will lower the observed foreshock rate (Jones, 1984). Second, these catalogs are certainly incomplete below magnitude 3 and may be incomplete below magnitude 4 . The completeness level is difficult to assess because the time period around larger earthquakes often received extra attention.

This larger catalog cannot be used to determine the overall rate of foreshock occurrence due to uncertainties in the mainshock focal mechanisms (Jones, 1984) but can shed light on the form of the magnitude distribution of foreshocks. Figure $2 \mathrm{c}$ shows a cumulative number plot of the number of events versus the difference in the mainshock and foreshock magnitudes. The theoretical distribution for the uniform distribution and log-normal distribution proposed by Lindh and Lim (1995) are also shown, under the assumption that the catalog is complete to a magnitude difference of 2 and that the cumulative distributions match exactly at that point. As above, we divided the data into 2, 3, 4, 5, and 6 equal-sized subsets and applied a Kolmogorov-Smirnoff one-sample test to determine if either distribution could be rejected by the data. When using two bins, the log-normal distribution is preferred because the flat section of the theoretical distribution from 0 to 0.5 magnitude units is in the same bin as the flat section in the data from 0.5 to 1 magnitude units. However, the uniform distribution cannot be rejected with two bins, even at the $80 \%$ confidence level. For three or more bins, the uniform distribution is preferred, but again, the log-normal distribution cannot be rejected. When using more than three bins, some of the bins are nearly empty, and thus the statistical tests have little power.

In addition to increasing the time period, we can also lower the magnitude threshold to enlarge our data set. Agnew and Jones (1991) used the southern California catalog since 1932 to produce a data set with $1510 \mathrm{M} \geqq 3$ mainshocks of which 669 have $M \geqq 2$ foreshocks. To avoid problems with catalog completeness, we use the $313 M \geqq 4$ mainshocks since 1945 of which 60 have foreshocks within 


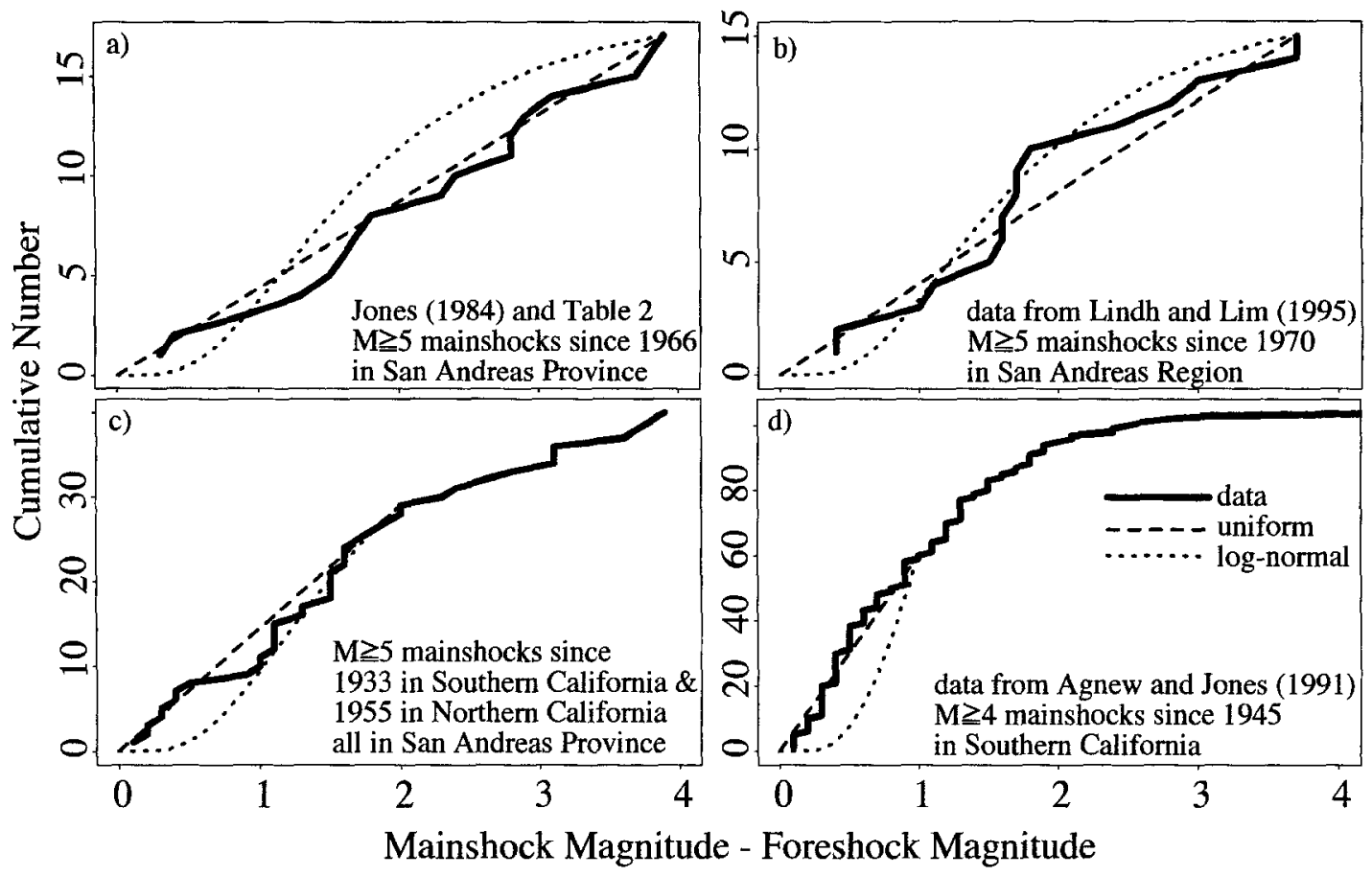

Figure 2. The cumulative number of foreshocks with respect to increasing magnitude difference between the mainshock and the foreshock. Also shown are the theoretical predictions of the uniform distribution and the log-normal distribution set to match at a point where the catalog is judged complete. (a) Data from Jones (1984) and Table 2. (b) Data from Lindh and Lim (1995). (c) 38 sequences with $M \geqq 5$ mainshocks and $M \geqq 2$ foreshocks. This data set includes events in the San Andreas physiographic province from the Caltech-USGS catalog from 1933 to 1994, the U.C. Berkeley catalog from 1950 to 1994, the USGS Northern California catalog from 1969 to 1994, Jones (1984), and the compilation shown in Table 2. Sequences with known dip-slip mainshock mechanisms were removed. (d) $M \geqq 4$ mainshocks from Agnew and Jones (1991) that had foreshocks within $10 \mathrm{~km}$ and 3 days.

one unit of magnitude (Fig. 2d). Data are only analyzed for foreshocks within one unit of their mainshock because the incompleteness of the catalog below magnitude 3 contaminates the data. Similar to the previous data set, this set may also include normal- and thrust-faulting events and so can only be used to investigate the shape of the magnitude distribution. Regardless of whether these data are divided into $2,3,4,5$, or 6 bins, the log-normal distribution can be rejected at above the $99.9 \%$ confidence level while the uniform distribution cannot be rejected at even the $80 \%$ confidence level. Only when six bins are used does one bin become almost empty, reducing the statistical power of the test. For this data set, we conclude that the uniform distribution provides an adequate fit to the data, but the log-normal distribution should be rejected.

Similar results can be obtained by limiting the data set to 1960 and later which yields 223 sequences of which 42 have foreshocks within one unit of magnitude. We note that for both starting times, $19 \%$ of the sequences have foreshocks within one unit of magnitude, above the rate predicted by Agnew and Jones (1991). For three units of magnitude difference, this would result in a $10 \%$ difference in the rate of foreshock occurrence, and, later, we will use this difference to compute ranges of possible results.

Jones et al. (1997) provide theoretical support for the uniform distribution. They modeled the magnitude distribution of foreshocks by assuming that the foreshocks are a subset of the aftershock process. This happens when an aftershock exceeds the size of the initial event in the sequence and becomes the mainshock, while the initial event becomes the foreshock. Using the Gutenberg-Richter relationship, they first show that the magnitude of the largest aftershock in a sequence, with respect to the initial event, is distributed like an asymmetric bell-shaped curve centered about one unit of magnitude below the initial event magnitude (Fig. 3). A few percent of the largest aftershocks have magnitudes greater than their initial event, and thus, these initial events are foreshocks, not mainshocks. The exact shape of this curve depends on the distributions of the $a$ - and $b$-values for aftershock sequences in that region.

The bell-shaped curve is the magnitude distribution of the largest event to follow an initial event, but the magnitude distribution of foreshocks with respect to their mainshocks is needed to use the formulation of Agnew and Jones (1991). 


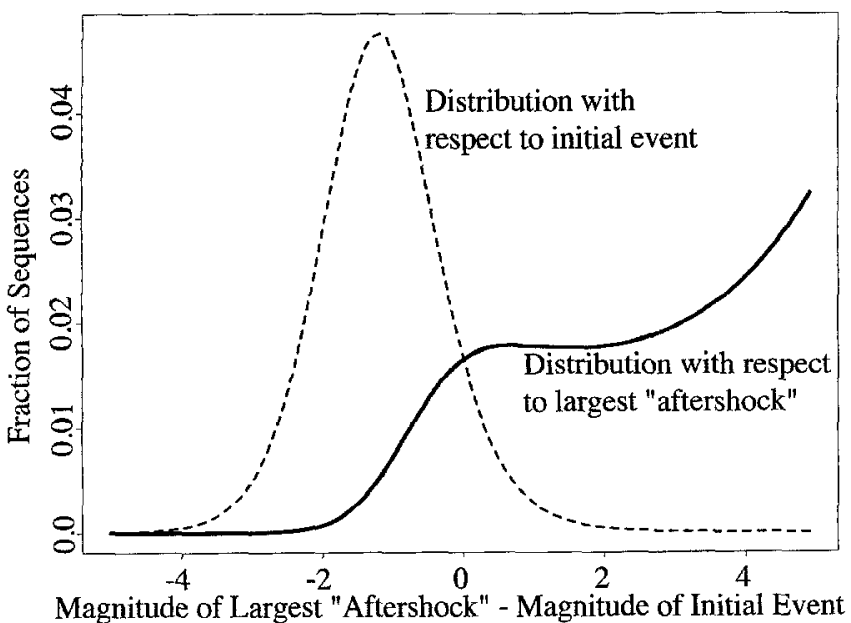

Figure 3. The predicted percentage of earthquake sequences as a function of the magnitude difference between the largest "aftershock" and the initial event of a sequence. We use "aftershock" in quotes to denote the largest event in a sequence, except for the initial event. The dotted line shows the distribution with respect to the initial event, and the solid line, with respect to the largest "aftershock." Positive values on the $x$ axis indicate foreshock-mainshock pairs, and negative values are mainshock-aftershock pairs. (after Jones et al., 1997). Curves are for California specific values with the distribution of aftershock $a$ and $b$-values from Reasenberg and Jones (1989), and $b=0.8$ for the declustered regional background seismicity.

This is the distribution of initial events with respect to the largest "aftershock" in a sequence. An $M 5$ event may be common in the aftershock sequence of an $M 7$ initial event and very unlikely after an $M 3$ initial event, but there are many more $M 3$ initial events than $M 7$ initial events. Thus, it may be more likely that a particular $M 5$ event will follow one of the many $M 3$ events in a region than it is to follow one of the relatively rare $M 7$ events in a region.

To model this, Jones et al. (1997) multiplied the theoretical distribution of aftershocks by the relative frequency of the initial events, which depends on the $b$-value for the declustered catalog. Again, the shape of the resulting distribution depends on the aftershock parameters $a$ and $b$, and on the regional $b$-value. If the regional $b$-value is close to the mean aftershock $b$-value, the two magnitude dependencies essentially cancel out, leaving a distribution of foreshocks relative to mainshocks that is approximately uniform with magnitude. In Figure 3, this is the essentially flat segment of the solid line for values from 0 to 3 . This curve predicts that $55 \%$ of the mainshocks in California should be preceded by a foreshock within three units of magnitude, which is in good agreement with the observations presented earlier.

We conclude that we do not yet have sufficient data to prove the magnitude distribution of foreshocks beyond all possible doubt. However, the log-normal distribution is pref- erable only when the data set is so small that no conclusive statements can be made. If we accept that earthquake selfsimilarity means that $M \geqq 4$ events do not behave in a way different than $M \geqq 5$ and $M \geqq 6$ events, we can conclude that the uniform distribution provides a significantly better fit than the log-normal distribution. This result, in addition to the theoretical support for the uniform distribution, leads us to conclude that the uniform distribution is preferable. However, because this can only be proven with data from mainshocks smaller than Parkfield, we will show the affect of this choice on the results.

Should we, however, use this generic distribution of foreshock behavior for strike-slip earthquakes in the San Andreas physiographic province for the Parkfield case? At Parkfield, half of the past four mainshocks had foreshocks with $M \geqq 5$, or within one unit of the mainshock's magnitude. Thus the observations at Parkfield suggest that this fault segment has more large foreshocks than other areas, but is this difference significant at the $95 \%$ confidence level?

Agnew and Jones (1991) assumes that $15 \%$ of the sequences should have foreshocks within one unit of mainshocks's magnitude based on the results of Jones (1984). Using this rate, and the binomial distribution, the probability of getting two or more $M \geqq 5$ foreshocks in the four sequences is 0.11 . Therefore, the confidence level that the Parkfield foreshock behavior is different than the average behavior is only $89 \%$. If $19 \%$ of the sequences should have foreshocks within one unit of magnitude, as in the $M \geqq 4$ data set, then the confidence level that Parkfield foreshock behavior is different from this slightly higher rate is only $84 \%$. Based on this analysis, we choose to apply the average rate of foreshock occurrence as determined by Agnew and Jones (1991) to the Parkfield case.

If one chooses to apply some higher rate based on the Parkfield history, the output alert probabilities will be higher; however, they will not be statistically justifiable at the $95 \%$ level. Moreover, making a distribution of foreshock rate versus foreshock magnitude will be difficult based on the small data set. The rate of foreshocks affects the result in a similar manner to the long-term mainshock probability. Hence, as discussed above, doubling or halving the rate of foreshocks would approximately double or halve the alert probabilities, respectively.

\section{Background Rate}

The final input needed to calculate the alert probabilities is the rate at which background events occur. This requires selecting the region in which earthquakes are considered possible foreshocks. Agnew and Jones (1991) required the foreshock epicenter to be within $10 \mathrm{~km}$ of the mainshock epicenter. Due to uncertainty in the expected mainshock epicenter, this required using the area within $10 \mathrm{~km}$ of the expected rupture. However, for Parkfield, the epicenters of the past two mainshock and foreshock hypocenters are well constrained to a small area under Middle Mountain (Bakun and McEvilly, 1979; Cole and Ellsworth, 1995). If only the 
mainshock hypocenter is expected to remain in the same spot, then the region used could be a $10-\mathrm{km}$-radius circle centered on the 1966 mainshock epicenter. If the foreshock is also expected to remain in the same spot, then an even smaller area could be used. Making these restrictions, based on the past observations, assumes that the rate of foreshocks may be the same as for other faults but that these foreshocks are confined to a smaller region.

The unusual character and behavior of the area around Middle Mountain supports the assumption that future Parkfield foreshocks will be confined to a smaller area. Not only did this region contain the previous two epicenters and foreshocks but it has displayed unusual sensitivity to stresses applied by remote sources such as the 1983 Coalinga earthquake (Poley et al., 1987). This may be explained by the high pore pressures that are inferred to exist from a combination of three-dimensional velocity models, gravity data, and electrical resistivity observations (Eberhart-Phillips and Michael, 1993) and a high $V p / V s$ ratio (Michelini and McEvilly, 1991).

While Middle Mountain has unusual characteristics and has been the initiation zone in the last two mainshocks, it may not be the initiation zone in the future. In the 1934 (Segall and Du, 1993) and 1966 (Segall and Harris, 1987) mainshocks, most of the moment was released from an area 8 to $25 \mathrm{~km}$ southeast of Middle Mountain (Fig. 4). This is the same area that Segall and Harris (1987) demonstrated was storing strain that could be released in a future event. It seems reasonable that the mainshock could be triggered by a hypocenter anywhere along the edge of the patch of stored strain and not just by one under Middle Mountain. In 1994, an $M 5.0$ earthquake occurred on the San Andreas fault between the Middle Mountain location of the 1934 and 1966 hypocenters and this region of primary moment release, suggesting that the two regions may not be as closely coupled now as when the previous larger earthquakes occurred. Moreover, while the last two, and probably three, mainshocks nucleated under Middle Mountain (Bakun and McEvilly, 1984), we do not know where the hypocenter was for the previous three events.

We simply do not have enough information about the complete earthquake history, the rupture process, the material properties along the fault, or the state of stress on the fault to come to a firm conclusion about where the next sequence will initiate. Given this uncertainty and our inability to demonstrate that Parkfield behaves in a significantly different way than the rest of California, we prefer to consider equally the possibility of foreshocks in the larger area used by Agnew and Jones (1991).

In order to illustrate the effect of making these choices, we will examine three possible regions: the area within 10 $\mathrm{km}$ of the expected rupture area (hereafter called the Park-

\section{Seismicity Along-Fault Cross-section 1982-1995}

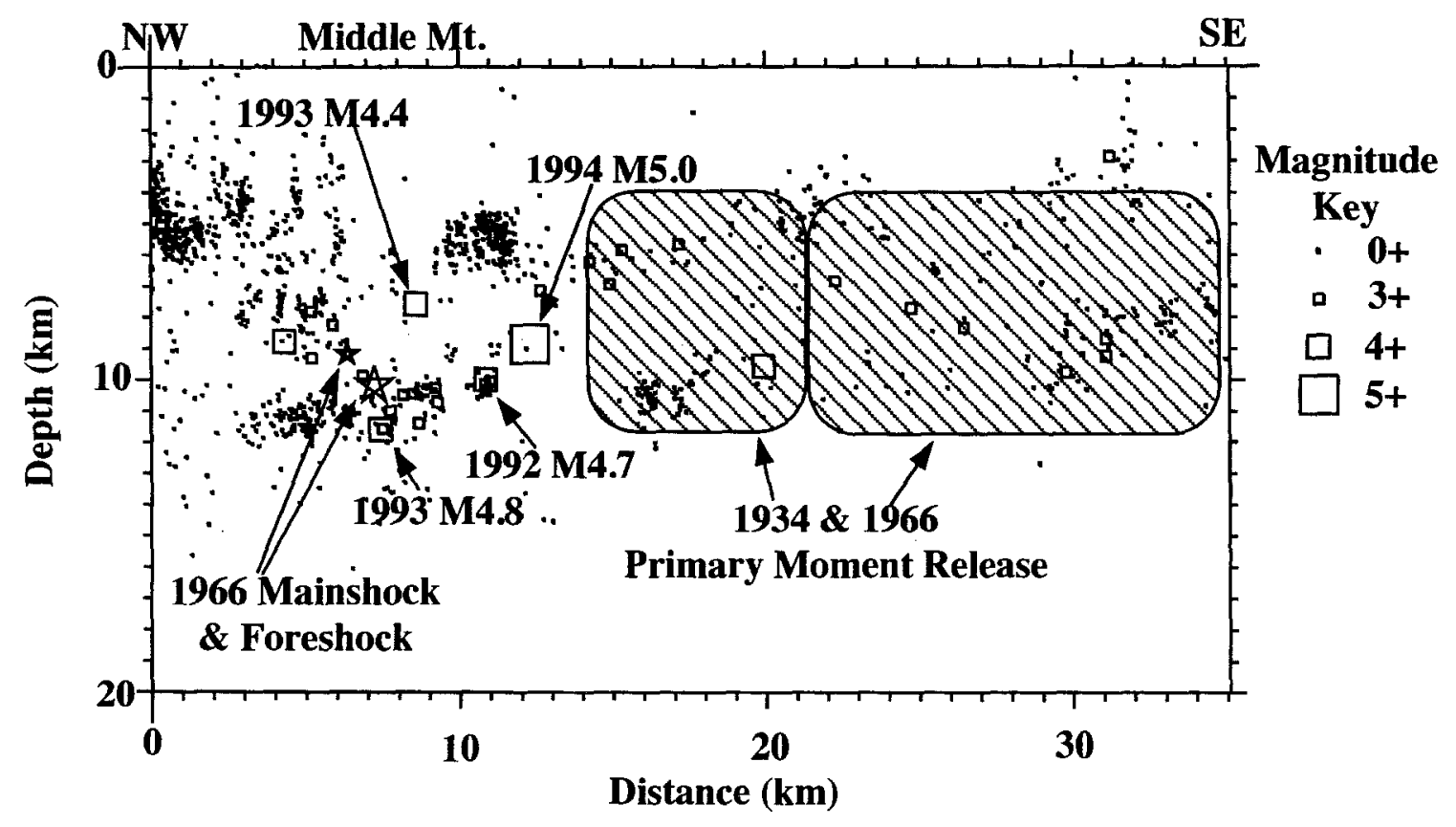

Figure 4. Along-fault cross section showing seismicity from 1982 (dots), on highlighting the 1966 mainshock and foreshock (stars), the $M \geqq 3$ events since 1992 (boxes), and the regions of primary moment release in the 1934 and 1966 mainshocks (hatched areas). 
field box), the original Middle Mountain box from OFR 87192 that approximately contains the area within $10 \mathrm{~km}$ of the 1966 hypocenter, and a Small Middle Mountain box containing the area within $2 \mathrm{~km}$ of the 1966 foreshock. Using the original Middle Mountain box, instead of a circle with a $10-\mathrm{km}$ radius centered on the 1966 mainshock epicenter, allows for a more direct comparison to the results in OFR 87192 , and because the seismicity is primarily located along the San Andreas fault, there will be little difference. An upper depth limit was placed on this box (OFR 87-192) to lower the background rate of earthquakes because the creeping segment of the San Andreas fault overlaps the Parkfield hypocenter at shallow depths. The Small Middle Mountain box illustrates the effect of assuming that all future foreshocks will be repeats of the 1934 and 1966 foreshocks. The 2-km half-width of this box was chosen based on uncertainties in the rapid earthquake locations.

To compute the background seismicity rate, the Northern California Seismographic Network catalog for the years 1982 through February 1995 was declustered by keeping only the largest events in \pm 3 -day windows. Starting in 1982, when the completeness level dropped to $M=1.2$ (Fig. 5 ), yielded more data, over a wider magnitude range, than using an $M=1.8$ cutoff since 1971 , as done in OFR $87-$ 192. This improves our ability to fit the data to the Gutenberg-Richter relationship. By declustering the catalog, we prevent the aftershock process from increasing the seismicity rate over the actual rate of independent background events. Agnew and Jones (1991) used the declustering method of Reasenberg (1985) to identify independent background events but did not, in addition, remove events that were smaller than another in their area within 3 days. This increases the background rate by including events that would not be considered as possible foreshocks. If we first used the declustering algorithm, and then removed events that are smaller than others in their alert box within 3 days, the size of the background catalog would decrease by $1 \%$ for the Parkfield box to $10 \%$ for the smaller boxes. This is much smaller than other uncertainties in the process, and the simpler approach chosen here is easier to apply in real time. If another larger event occurs before the 3-day window is over, the current alert will either be extended at the current level or moved to a higher alert level. This means that when seismicity increases in magnitude over time, alerts will be declared based on events that would not be in the declustered catalog. However, these alerts will generally be low-level ones.

We could have declustered the catalog by only removing events that had a larger one in the 3 days before them, but then the background catalog would not correspond well to the chosen foreshock distribution. The foreshock distribution with respect to magnitude is based on only the largest event in the 3 days before the observed mainshocks. We feel it is more important for the background distribution to correspond to the foreshock distribution than to avoid slightly underpredicting the frequency, and slightly overpredicting

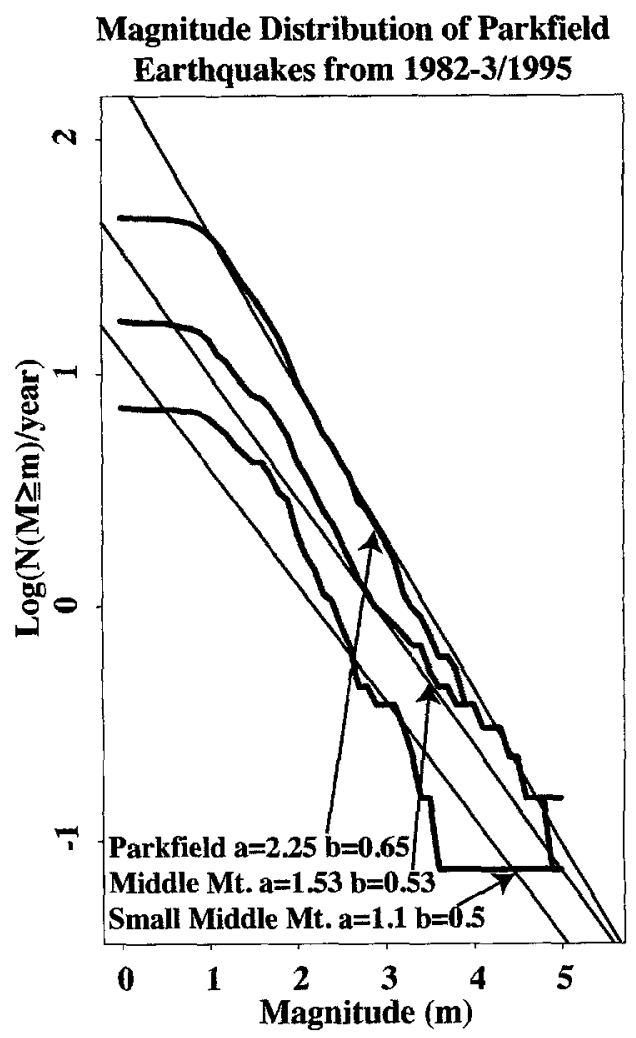

Figure 5. Cumulative seismicity plots versus magnitude for the three alert boxes considered for the time period from 1982 through February 1995.

the probability, of the lower-level alerts. We tested the affect of making this choice on the alert probabilities, and it is on the order of $10 \%$. It also tends to cancel the effect of not using the Reasenberg (1985) declustering algorithm. Thus, these effects are small compared to the other possible errors in the data and assumptions.

Distributions of the declustered seismicity rate versus magnitude are shown in Figure 5 for three possible boxes: our preferred Parkfield box, the original Middle Mountain box, and the Small Middle Mountain box (Fig. 1 and Table 2). For the two larger boxes, the linear fits to the distributions were determined by using a maximum likelihood method (Aki, 1965). The Middle Mountain box is a subset of the Parkfield box; therefore, its $a$-value was decreased slightly to keep the entire distribution below that for the Parkfield box.

For the Small Middle Mountain box, the observed distribution departs too far from a straight line to obtain a satisfactory fit by this objective method. To fit this distribution, we first constrained the activity at the largest magnitude by using the longer-term catalog compiled for $M \geqq 3.7$ events by Cole and Ellsworth (1995). In the Small Middle Mountain box, since 1930, their catalog includes the 1934 and 1966 foreshocks and mainshocks, and $M=4.8$ events on 5 June 1934, 28 December 1939, 16 November 1956, and 14 November 1993. The last three occurrences of this event are 
Table 2

Vertices of Regions

\begin{tabular}{lccl}
\hline \multicolumn{1}{c}{ Region } & Depth $(\mathrm{km})$ & Latitude $(\mathrm{N})$ & Longitude $(\mathrm{W})$ \\
\hline San Andreas Fault & $\leqq 20$ & 3558.26 & 12035.86 \\
(mainshock definition) & & 3601.74 & 12030.74 \\
& & 3547.25 & 12015.72 \\
Parkfield & & 3543.77 & 12020.82 \\
& all & 3556.53 & 12038.41 \\
& & 3603.47 & 12028.19 \\
Middle Mountain & & 3548.98 & 12013.17 \\
& $\geqq 6.5$ & 3542.04 & 12023.37 \\
& & 3557.5 & 12029.5 \\
Small Middle Mountain & $\geqq 7.5$ & 3552.0 & 12025.0 \\
& & 3556.0 & 12038.0 \\
& & 3559.45 & 12030.42 \\
& & 3557.73 & 1203038.10 \\
& & & 12032.14 \\
\hline
\end{tabular}

in the background classification and were the only ones used to measure the background rate over this time period. This gives a rate of occurrence of $0.046 M \geqq 4.8$ events per year in the Small Middle Mountain box. We constrained the linear fit to intersect this point and then tested slopes from $b=0.3$ to $b=0.8$. A slope of $b=0.5$ fits the data decently, especially at the higher-magnitude levels where the most important alerts will occur.

However, the difficulty fitting a straight line to the data in the Small Middle Mountain box illustrates another problem with using such a small volume for the alert system. The magnitude distribution for background seismicity used by Agnew and Jones (1991) is based on applying the linear fit to the cumulative magnitude distribution of the background seismicity. With such a small box, this is difficult and leads to higher uncertainties in the alert probabilities.

The maximum likelihood calculations gave average uncertainty in the $b$-values of \pm 0.075 . Comparison of the data to the Gutenberg-Richter relationship using this uncertainty in $b$-value suggests that the uncertainty in the $a$-values is about \pm 0.2 . These values will be used when computing uncertainties in the alert probabilities.

\section{Mainshock Definition}

We can now compute the probability that an earthquake will be followed by the Parkfield mainshock. But to know if this prediction has been fulfilled, we must define the expected mainshock. In OFR 87-192, the Parkfield mainshock is defined to be a magnitude 6 earthquake along the San Andreas fault near Parkfield, California. However, the magnitude of past Parkfield earthquakes have not been exactly 6, and in OFR 87-192, a sample warning message modifies this to be "about 6 ." This is a vague definition because some of the terms are loosely defined such as "about 6," "near Parkfield," and, in light of the 1989 Loma Prieta earthquake, even the term "along the San Andreas fault" can be open to interpretation.

The long-term probability is an input to the alert probabilities. Therefore, the definition of the expected mainshock should correspond to the catalog of events used to determine the long-term probability. As one goes back in time, less and less is known about the individual mainshocks in the Parkfield catalog. The teleseismic records show that the last three are all about moment magnitude 6. Within the errors cited by Bakun and McEvilly (1984), all three have $M w \geqq 5.7$. All six events are known to have produced surface rupture along the San Andreas fault system (including the southwest fracture) near Parkfield (Bakun, 1988). Thus, we define the Parkfield mainshock to be an event with $M w \geqq 5.7$ that produces surface rupture along the San Andreas fault, and/or the Southwest Fracture zone, between $36^{\circ} \mathrm{N}$ and $35^{\circ} 45^{\circ} \mathrm{N}$ and within $5 \mathrm{~km}$ of the mapped trace. Coordinates of this box are shown in Table 2, and it is the same length, but half the width, of the Parkfield box shown in Figure 1. Events that produce additional surface rupture outside of this box or on other faults are also Parkfield mainshocks. We do not have a strong preference for the source of the $M w$ determination. The Harvard CMT catalog, the University of California at Berkeley catalog, and the Caltech-USGS catalog are all reasonable candidates.

We choose to use the surface rupture to describe Parkfield mainshocks because it is a known characteristic of the past six Parkfield mainshocks and because of problems with two other possible measures: the mainshock hypocenter and the centroid of the moment release. A hypocenter near the edge of a Parkfield box could release most of its moment elsewhere. Therefore, using the hypocenter to define the mainshock could lead to us defining an event as a Parkfield event while not believing it is really a repeat of the previous events. A moment tensor centroid would be an improvement, but it is possible that location errors in these determinations would provide a wrong answer until long after the mainshock occurs. If accurately located (errors of less than $5 \mathrm{~km}$ ) moment tensor centroids could be determined quickly, they may be a better choice than using the surface rupture.

\section{Results}

Given the preferred assumptions discussed earlier, we can compute the probability that an observed event is a foreshock and that therefore the mainshock will follow within the next 3 days. Figure 6 illustrates these probabilities for the three alert boxes under consideration, with a comparison to the values from OFR 87-192.

For the Middle Mountain box, for which probabilities have been computed using both methods, the new methodology gives probabilities that are lower than OFR $87-192$ by a factor of 2.5 to 5 with the biggest difference for events between $M 4$ and $M$ 5. If the Parkfield box that we prefer is used with the new methodology, then the difference with the OFR 87-192 result is as large as a factor of 14 , with differ- 


\section{Parkfield Alert Probabilities}

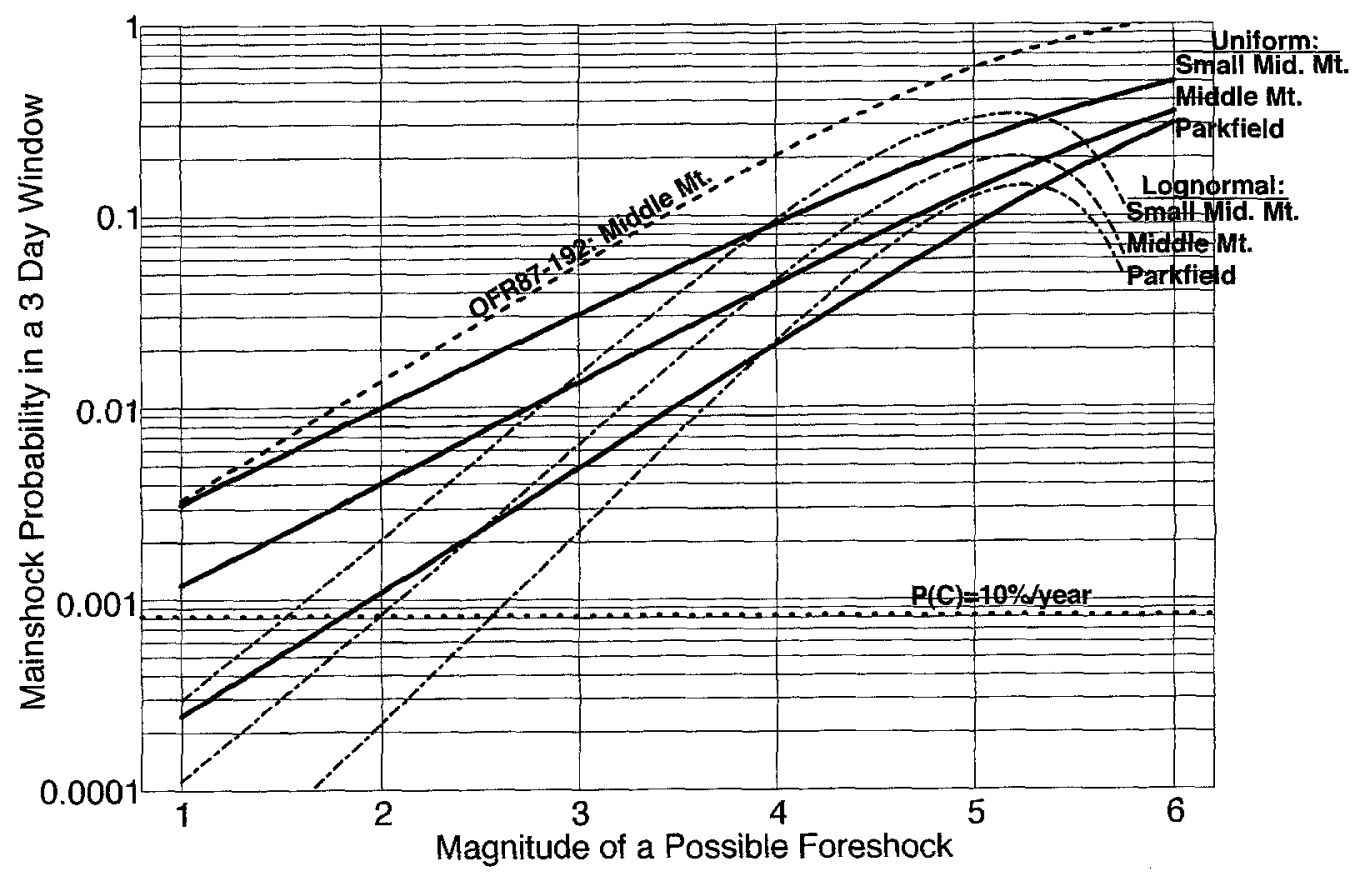

Figure 6. Probabilities of the mainshock occurring within the 3 days after a possible foreshock occurs. Curves are shown for the three alert boxes considered with both the uniform (solid line) and log-normal (dashed and dotted line) distribution of foreshock magnitudes, the results of OFR 87-192 (dashed line), and the 10\%/year value for the long-term mainshock probability (dotted line).

ences between $M 4$ and $M 5$ about a factor of 7 to 10 . Thus, the difference is large in the magnitude range most important for public policy considerations.

The values determined in this report are lower than those in OFR 87-192 for several reasons: the lower longterm probability of the mainshock occurring used here, the lower rate of foreshocks assumed for each mainshock, and the new methodology used. Another factor is that since October 1992, the Parkfield area has increased in activity, especially at the higher magnitude levels. Including these data into our estimate of the background seismicity rates has increased these rates and therefore decreased the probability that any event is a foreshock.

To produce alert probabilities for the log-normal distribution requires giving the mainshock magnitude, which for Parkfield is taken to be $M 6$, and an uncertainty in the magnitudes, which Lindh and Lim (1995) set at 0.25. The lognormal distribution of foreshock magnitude produces results that are similar to the uniform distribution, except that they have a greater value near the mode of the log-normal distribution, 1.1 units below the mainshock magnitude, and the log-normal results are lower than the uniform results both above and below this region. It is not possible to produce results for the log-normal distribution when the possible foreshock magnitude exceeds the mainshock magnitude minus the magnitude uncertainty; however, this is not a prac- tical problem because at that point the candidate event may well fit the definition of the mainshock.

Perhaps more important than the differences between the old and new results, and between the different foreshock magnitude distributions, is the range of results possible given the various possible assumptions and uncertainty in the various seismicity and foreshock rates. In Figure 7, the lines labeled "Max. All Regions" and "Min. Both" show the largest and smallest alert probabilities that can be obtained under the following sets of conditions: the mainshock probability from $4 \%$ to $10 \%$ per year, the probability of foreshocks before mainshocks from $40 \%$ to $60 \%$, the magnitude distribution of foreshocks with respect to the mainshock taking either the uniform or log-normal distribution, and using any of the three boxes with uncertainty in the $b$-value of \pm 0.075 and in the $a$-value of \pm 0.2 . The range produced in these calculations is a plausible limit on the results that could be obtained under assumptions currently under debate. They are, however, not confidence limits on the results because they include choices of discrete assumptions and we cannot assign probabilities to these choices. If we could compute $95 \%$ confidence limits, they would be smaller than the range shown.

Given these uncertainties, the range between the upper and lower bounds is very large. For a potential foreshock with $M=4$, the probabilities could range from $0.6 \%$ to 


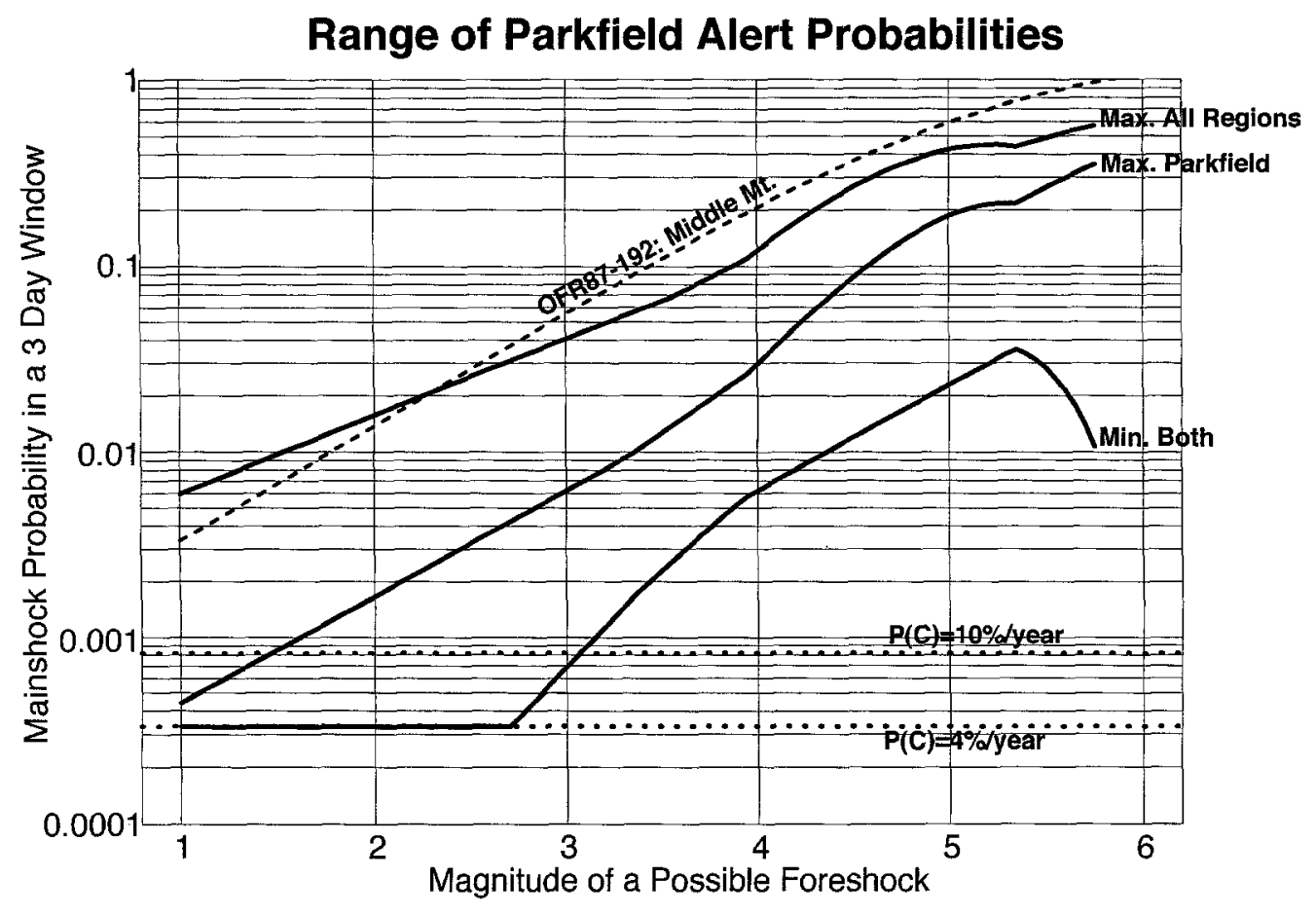

Figure 7. Range of possible probabilities of the mainshock occurring within the 3 days after a possible foreshock occurs (solid lines). "Max. All Regions" considers the results for all three possible alert areas. "Max. Parkfield"' considers only the Parkfield alert area. "Min. Both" is the same for either all three alert areas or only the Parkfield area. The results from OFR 87-192 (dashed line) are shown for comparison. The $10 \%$ /year and $4 \%$ /year values for the long-term mainshock probability are also shown (dotted lines).

$12 \%$, at $M=5$ the range is from $2.2 \%$ to $42 \%$. Much of this range comes from the choice of three possible foreshock regions. If only the Parkfield region is considered, then the maximum value is shown in Figure 6 by the line labeled "Max. Parkfield". In this case, for an $M 4$ potential foreshock, the range of probabilities is from $0.6 \%$ to $3 \%$, and for $M 5$, from $2.2 \%$ to $19 \%$.

We do, however, note that except for the smallest possible foreshocks, the minimum value is much greater than the long-term probability of the mainshock occurring on its own. Thus, the results show that earthquakes cluster, and this fact produces significant short-term increases in the probability of future events, but it may be difficult to quantify that increase.

\section{Discussion}

There are two issues to discuss. First, given the new alert probability calculations, how could the seismicity alert structure at Parkfield be changed? This first part of the discussion will use only the uniform distribution for the magnitude distribution of foreshocks because it is preferred both on observational and theoretical grounds. Second, given the uncertainties in making these calculations, is having an alert structure useful? For this second section, we will include the log-normal distribution for foreshocks.

The probability of the mainshock occurring in a 3-day window associated with an A-level alert in OFR 87-192 is at least $37 \%$. With our results, this level cannot be reached in either the Parkfield or Middle Mountain boxes. For the small Middle Mountain box and the uniform distribution, it would require an $M 5.7$ event, large enough that it would fit our definition of the mainshock if it produces ground rupture. The lowest magnitude that could trigger an A-level alert under OFR 87-192 is 4.5 . At this magnitude, the new formulation would give a probability of only $4 \%$ for the Parkfield box, $8 \%$ for the Middle Mountain box, and $15 \%$ for the Small Middle Mountain box. If we continued to associate $M 4.5$ events with A-level alerts, then the meaning of the alert levels has changed. We therefore do not suggest keeping the same alert rules with respect to magnitude and only changing the associated probabilities. To do so would destroy the communication value of the words "A-level alert." We believe this should be reserved for cases where the associated probabilities are high enough to warrant action on the part of groups concerned with earthquake response.

One possibility is to use the structure set up for southern California (Jones et al., 1991). There, D-level alerts occur when the probability of a mainshock occurring in the next 
3 days reaches $0.1 \%$. Higher-level alerts include $\mathrm{C}$ level at $1 \%$ and $\mathrm{B}$ level at $5 \%$. The B level is stated to extend from $5 \%$ to $25 \%$, and they did not implement the A level that would occur above $25 \%$. For Parkfield, we will use A level when the probability exceeds $25 \%$. For the three boxes, this would result in the alert criteria for single foreshocks shown in Table 3.

Two points should be noted about this alert structure. First, it is only possible to reach an A-level alert if the Small Middle Mountain box is used. However, we emphasize that using such a small box is only valid if events evolve exactly as they did in 1934 and 1966. In our opinion, this assumption is unjustified. This is why OFR 87-192 included C- and Dlevel alerts for a larger Parkfield box. And even for the Small Middle Mountain box, a repeat of the 1934 or 1966 foreshock would only be an A-level alert if the preliminary magnitude is not underestimated. We also note that the threshold to get to a D-level alert is lower than the USGS Northern California Seismic Networks completion level for the Middle Mountain box and below its detection threshold for the Small Middle Mountain box. This presents an operational problem.

W. Ellsworth (personal comm., 1994) has suggested an alert scheme that would require a repeat of the $M 5$ foreshock that occurred 17 min before the 1934 and 1966 mainshocks, as determined through waveform cross-correlation, to go to an A-level alert. An event with this magnitude and location has only been observed twice and both times it was a foreshock, hence it is impossible to assess the background rate for this event, and therefore, it is not meaningful to assess the alert probability associated with it. If this event is never a background earthquake and always a foreshock, then the alert probability should be $100 \%$, although our confidence in this value should be low because it is based on only two observations. Perhaps to implement such an alert scheme requires developing a much deeper physical understanding of the foreshock process than now exists. Until that happens, we must rely on statistical analyses. Given that there are only three well-recorded repeats of the Parkfield earthquake sequence, and the mainshock catalog includes three events that we know very little about, we prefer to avoid making specific assumptions based on the uniqueness of the 1934 and 1966 sequences. Instead, we suggest using the larger Parkfield box as the new alert boundaries for seismicity.

Instead of using a single alert box, one could use multiple boxes in the alert scheme, for example, the Small Middle Mountain box and the Parkfield box. To use these two boxes properly, you have to assign a probability, called $q$, that the foreshock, if one occurs, will occur in the Small Middle Mountain box. Then the probability of a foreshock in the Small Middle Mountain box is the product of $q$ and the generic foreshock distribution. The alert probabilities would have to be recomputed for the Small Middle Mt box based on this new foreshock distribution and will be lower than shown in Figure 5 and above. Then the remaining
Table 3

Foreshock Alert Structure

\begin{tabular}{ccccc}
\hline & & \multicolumn{3}{c}{ Magnitude Required for Level } \\
\cline { 3 - 5 } Alert Level & $\begin{array}{c}\text { Mainshock } \\
\text { Probability }\end{array}$ & Parkfield & Middle Mountain & $\begin{array}{c}\text { Small Middle } \\
\text { Mountain }\end{array}$ \\
\hline A & $25 \%$ & 5.9 & 5.7 & 5.1 \\
B & $5 \%$ & 4.6 & 4.2 & 3.5 \\
C & $1 \%$ & 3.5 & 2.8 & 2.1 \\
D & $0.1 \%$ & 2.0 & 0.9 & 0.0 \\
\hline
\end{tabular}

chance that a foreshock will occur, 1-q, is assigned to the larger Parkfield box that will also now have a foreshock distribution lower than the generic one, and therefore, the alert probabilities will also decrease. However, the background rate for the larger box must be decreased by the rate of earthquakes in the Small Middle Mountain box. This latter effect will increase the alert probabilities by some amount that depends on the relative rates of activity between the two boxes.

While we suggest a method for using multiple alert boxes, we have chosen not to show an example because we do not know how to set the probability, $q$, or which boxes should be used. One could even extend this method to use all three boxes. Ideally, these choices should be made independent of the final results. We are therefore reluctant to show an example. Moreover, we emphasize that implementation of this scheme would require making assumptions that cannot be verified.

So far, we have focused on the best-fit values for the three boxes using the uniform distribution. If we instead look at the range of possible values, a problem with this alert scheme appears. Depending on our choice of assumptions, a magnitude 5 event that occurs inside the Small Middle Mountain box could yield alert probabilities from $2.2 \%$ to $42 \%$, which could give an alert level of $\mathrm{C}, \mathrm{B}$, or A. If we use only the Parkfield box for our interpretation, the alert probabilities range from $2.2 \%$ to $19 \%$, which could give an alert letter of either $\mathrm{C}$ or $\mathrm{B}$. Hence, for the events of greatest interest, the uncertainty is large enough that it is difficult to assign an alert level. While OFR 87-192 stated that the alert probabilities were too uncertain to quote to two significant digits, with errors as large as a factor of 10 to 20 , we question whether they should be quoted to even one significant digit.

As shown above, much of the uncertainty comes from the choice of which box to use. This is a debate that can presently occur only for the Parkfield segment. Nowhere else do we have sufficient knowledge to discuss limiting the foreshock region to a subset of the segment length. It is ironic that the extra knowledge we have for the Parkfield area results in more uncertainty. Does this uncertainty exist for other segments except that the available information is too sparse to start the debate? Or does the extra, but perhaps still small, amount of knowledge that exists for the Parkfield segment lead us into debating unwarranted assumptions? 
Given these large uncertainties, one must question the wisdom of the alert structure. Our knowledge of earthquake clustering is certain enough that we know that an $M \geqq 3$ Parkfield event raises the odds that mainshock will occur during the next 3 days as compared to the background rate (Fig. 6). But the range of uncertainties is too large to make meaningful use of the current alert structures. Refining the alert structure, given these uncertainties, requires input from the emergency response community and other users. We need to know at what probabilities they would take actions and how much uncertainty they can accept in these probabilities.

\section{Conclusions}

We have reevaluated the probability that observed earthquakes at Parkfield will be foreshocks to the Parkfield mainshock. This reevaluation includes reducing the longterm probability of the mainshock occurring, decreasing the rate at which foreshocks are assumed to precede Parkfield mainshocks, and using the methodology of Agnew and Jones (1991). Further, we suggest using the Parkfield box as the alert area in which foreshocks are assumed to occur because of uncertainties in how the first three Parkfield earthquakes sequences occurred and how the next Parkfield earthquake sequence will unfold. The $M \geqq 5$ mainshock catalog of Jones (1984) has been updated, and these results confirm her conclusions about the rate of foreshocks before strike-slip earthquakes in the San Andreas physiographic province.

The alert probabilities computed with this new formulation are lower than those in OFR 87-192, and thus revision of that document is required. This was partially done in 1995, but the interim plan that was adopted included probabilities calculated by Lindh and Lim (1995) who used a log-normal function to describe the distribution of foreshock magnitudes. Both observational and theoretical arguments presented here suggest that this is inappropriate. The interim plan also uses both the Small Middle Mountain and Parkfield alert boxes proposed above but ignores the earlier discussion and assigns all of the foreshock rate to both boxes. Finally, it includes W. Ellsworth's (personal comm., 1994) suggestion that waveform correlation be used to identify repeats of the 1934 and 1966 foreshocks in order to reach an A-level alert despite our inability to compute an alert probability associated with such an occurrence. Thus the alert probabilities in the new interim plan are too high, and a new alert structure for Parkfield seismicity alerts is still needed. For uniformity, we suggest using one similar to that adopted for southern California. Here the probability that any earthquake will be followed by the mainshock is calculated, and an alert is then declared if the probability exceeds a given value ( $25 \%$ for $\mathrm{A}, 5 \%$ for $\mathrm{B}, 1 \%$ for $\mathrm{C}$, and $0.1 \%$ for $\mathrm{D}$ ).

Before simply formulating a new alert structure for Parkfield, we suggest that more discussion focus on the uncertainty in these probability calculations. Given the large ranges of results that can be obtained by taking different sets of assumptions and the statistical errors associated with some of the input parameters, it is possible that the emergency services community and other users will find these numbers difficult to use. One could even question the societal value of quoting such an uncertain result.

\section{Acknowledgments}

This article has benefited from reviews and comments from Duncan Agnew, Bill Bakun, Bill Ellsworth, Lind Gee, Dave Jackson, Allan Lindh, Paul Reasenberg, Jim Savage, and an anonymous reviewer.

\section{References}

Agnew, D. C. and L. M. Jones (1991). Prediction probabilities from foreshocks, J. Geophys. Res. 96, 11959-11971.

Aki, K. (1965). Maximum-likelihood estimate of $b$ in the formula $\mathrm{N}=\mathrm{a}$ $10^{-\mathrm{bM}}$ and its confidence limits, Bull. Earthquake Res. Inst. 45, 237239.

Bakun, W. (1988). History of significant earthquakes in the Parkfield area, Earthquakes and Volcanoes, 20, 45-51.

Bakun, W. H. and A. G. Lindh (1985). The Parkfield, California, earthquake prediction experiment, Science 229, 619-624.

Bakun, W. H. and T. V. McEvilly (1979). Earthquakes near Parkfield, California: comparing the 1934 and 1966 sequences, Science 205, 13751377.

Bakun, W. H. and T. V. McEvilly (1984). Recurrence models and Parkfield, California, earthquakes, J. Geophys. Res. 89, 3051-3058.

Bakun, W. H., K. S. Breckenridge, J. Bredehoeft, R. O. Burford, W. L. Ellsworth, M. J. S. Johnston, L. Jones, A. G. Lindh, C. Mortensen, R. J. Mueller, C. M. Poley, E. Roeloffs, S. Schultz, P. Segall, and W. Thatcher (1987). Parkfield, California, Earthquake prediction scenarios and response plans, U.S. Geol. Surv. Open-File Rept. 87-192, 45 pp.

Cole, A. T. and W. L. Ellsworth (1995). Earthquake clustering and the longterm evolution of seismicity near Parkfield, California, 1931-1994 (abstract), Seism. Res. Lett. 28.

Eberhart-Phillips, D. and A. J. Michael (1993). Three-dimensional velocity structure, seismicity, and fault structure in the Parkfield region, central California, J. Geophys. Res. 98, 15737-15758.

Jones, L. M. (1984). Foreshocks (1966-1980) in the San Andreas system, California, Bull. Seism. Soc. Am. 74, 1361-1380.

Jones, L. M. et al. (1991). Short term earthquake hazards assessment for the southern San Andreas fault, U.S. Geol. Surv. Open-File Rep. 91$32,29 \mathrm{pp}$.

Jones, L. M., R. Console, F. diLuccio, and M. Murru (1995). Are foreshocks mainshocks whose aftershocks happen to be big?, Eos. 76 (abstract), 388.

Lindh, A. G. and M. R. Lim (1995). A clarification, correction, and updating of Parkfield, California, earthquake prediction scenarios and response plans (USGS Open-File Report 87-192), U.S. Geol. Surv. Open-File Rep. 95-695, 39 pp.

Michael, A. J. and L. M. Jones (1995). A Reevaluation of the seismicity alert probabilities at Parkfield, California, U.S. Geol. Surv. Open-File Rept. 95-630, 24 pp (also available via http:/quake.wr.usgs.gov/ $\sim$ michael/ofr95-630).

Michelini, A. and T. V. McEvilly (1991). Seismological studies at Parkfield, I: Simultaneous inversion for velocity structure and hypocenters using cubic b-splines parameterization, Bull. Seism. Soc. Am. 81, 524552.

NEPEC Working Group to Evaluate the Parkfield Prediction Experiment (1993). Earthquake research at Parkfield--1993 and Beyond, U.S. Geol. Surv. Open-File Rept. 93-622, 19 pp. 
Poley, C. M., A. G. Lindh, W. H. Bakun, and S. S. Schulz (1987). Temporal changes in microseismicity and creep near Parkfield, California, $\mathrm{Na}$ ture 327, 134-137.

Reasenberg, P. (1985). Second-order moment of Central California seismicity, 1969-1982, J. Geophys. Res. 90, 5479-5496.

Reasenberg, P. A. and L. M. Jones (1989). Earthquake hazard after a mainshock in California, Science 243, 1173-1176.

Savage, J. C. (1995). The Parkfield prediction fallacy, Bull. Seism. Soc. Am. $83,1-6$.

Segall, P. and Y. Du (1993). How similar were the 1934 and 1966 Parkfield earthquakes?, J. Geophys. Res. 98, 4527-4538.

Segall, P. and R. Harris (1987). Earthquake deformation cycle on the San Andreas fault near Parkfield, California, J. Geophys. Res. 92, 1051110525.
Zoback, M. L. and M. D. Zoback (1980). State of stress in the conterminous United States, J. Geophys. Res. 85, 6113-6156.

U.S. Geological Survey

345 Middlefield Road-MS 977

Menlo Park, California 94025

(A. J. M.)

U.S. Geological Survey

525 So. Wilson Avenue

Pasadena, California 91106

(L. M. J.)

Manuscript received 5 November 1996. 